\title{
Bus Transport Process Networks with Arbitrary Launching Times
}

\author{
Zsolt Ercsey $^{1}$, Albert Nagy $^{2}$, József Tick ${ }^{3}$, Zoltán Kovács ${ }^{4}$ \\ ${ }^{1}$ Department of System and Software Technology, Faculty of Engineering and \\ Information Technology, University of Pécs, Boszorkány u. 2, 7624 Pécs, \\ Hungary, e-mail: ercsey@mik.pte.hu \\ ${ }^{2}$ Doctoral School of Applied Informatics and Applied Mathematics, Óbuda \\ University, Bécsi út 96/b, 1034 Budapest, Hungary, e-mail: albert.nagy@me.com \\ ${ }^{3}$ John von Neumann Faculty of Informatics, Óbuda University, Bécsi út 96/b, \\ 1034 Budapest, Hungary, e-mail: tick@uni-obuda.hu \\ ${ }^{4}$ Optin Ltd. Oroszlán u. 4, 6720 Szeged, Hungary, \\ e-mail: zoltan.kovacs@optin.hu
}

\begin{abstract}
The current paper is about a process network synthesis solution for a bus transport problem, where arbitrary launching data are given in an available timetable. Here, the bus transport problem is presented as an application of the p-graph methodology and is investigated from structural point of view. Focusing on the synthesis step, the bus transport process network is generated and detailed. Based on the maximal structure of the problem a mathematical programming model is generated which has the advantage of a smaller number of variables and constraints than the conventional mathematical models for similar problems. The solution of the mathematical programming model, results in the optimal solution of the bus transport problem. From the traffic point of view, an example of medium difficulty is solved, by a publicly available solver.
\end{abstract}

Keywords: optimization; mathematical programming model; process network; p-graph; synthesis; bus transport

\section{Introduction}

Sustainable urban mobility demands significant attention for effective operations. Optimization of public transportation contributes to an attractive and healthy urban environment, while further improving overall competitiveness. The industry has seen major technological advancements over the last decade: Buses represent more than just a way to get from one destination to another. Emerging new technologies, automation of processes, car sharing and other factors influencing 
public transportation habits also rapidly and continuously alter situations. It is of high importance that public transport service companies exploit their resources at the maximum level, while focusing on the most effective service availability for their passengers.

The rapid adoption of telematics systems at bus operators has extended the possibilities of the historically available user generated data which improved the monitoring of services, further motivating the operators to enhance daily operations. These data can be used both at the strategic planning and the tactical planning, considering frequency setting; robust timetable design as well as vehicle and crew scheduling. Optimizing this as a whole is an immensely complex issue, even its subtasks may lead to NP hard problems. Until recent past, solution of even small subproblems often met computational difficulties. The dynamic development of the technology, and the continuous improvement of the methodologies and models, together with the applied solution algorithms, make it now possible to solve real size, real case problems.

In this paper operational optimization issues are considered, where bus transport service companies usually have more remarkable influence. Since operational costs cover the main part of the expenditures of these service companies, any savings in this regards have great leverage. It is under their direct control, how their bus fleet is scheduled, how their drivers are assigned to the various work shifts both in the short and in the long run. In general, this problem involves legal perspective, employee interests, as well as individual claims. The constraints correspond to international and national regulations as well as company specific standards in terms of working hours, driving times, breaks or rest periods, depot considerations, labor availabilities etc. It is obvious, that each bus transport service company has its own specific expectations and constraints due to its particular business situation, which had to be handled together with common considerations. The decision support system suggested here is based on the p-graph methodology. The current method was developed and presented for the Budapest Transport Corporation, from where the published example originates. The purpose of the work was to effectively solve a vehicle and driver scheduling task that can be injected into the company's available information system. The paper is structured as follows. In Section 2 a literature review on vehicle scheduling problems and on the p-graph methodology is followed by the problem definition. In Section 3 the proposed solution framework is presented and detailed, namely (i) the maximal structure of the bus transport synthesis problem with arbitrary launching times is presented, the key elements of the $i$-th launch is highlighted and discussed, (ii) the mathematical programming model that suits the particular maximal structure is presented and (iii) is followed by an example. Section 4 presents conclusions for the most important results. Based on the synthesis step, the presented method has the advantage of being limited in the number of variables and constraints and therefore publicly available solvers are capable of generating results of real size problems. 


\section{Materials and Methods}

\subsection{Vehicle Scheduling Problems}

Public transport scheduling problems are very complex, most of them are NPcomplete when examining them from a theoretical point of view. Frequently, the proposed models are suggested for selected situations considering rules relating to timetabled and overhead trips, bus types and capacities. Commonly, it is not possible to include other conditions that arose in other real application environments. Several papers distinguish the problems by the number of device types and the number of depots. For example, the Single Depot Vehicle Scheduling Problem (SDVSP), where the vehicles belong to a single vehicle type and are located in the same physical location, and the Multiple Depot Vehicle Scheduling Problem (MDVSP), which considers different scheduled trips (and their vehicles) with different special needs. Alternative-fuel vehicles are also gaining advantage, which implies further research, thus assigning the available fleet of vehicles to service the given set of trips with specific start and end times.

The heart of these methods is the mathematical programming model, e.g. MILP or MINLP, which supposedly ensures the optimality of the resultant solution. Their algorithmic generation is a common step in most of the papers and is rarely detailed. Since the crucial difference is in the underlying mathematical programming models, these are elaborated and illustrated. Nevertheless, it is often difficult to generate and solve the complete models.

Bodin et al. [1] summarizes routing and scheduling problems of vehicles and crews. It classifies and categorizes routing and scheduling problems, as well as reviews algorithmic techniques and solution methodologies. The applicability of the results for bus transport problems is limited, however. Time-space network flow model is suggested in Kliewer et al. [2] involving multiple depots for vehicles and different vehicle types for bus scheduling. Integer programming methods with heuristic solution techniques are proposed by Dávid and Krész [3] and Tóth and Krész [4]. More recently a branch-and price based solution is suggested by Horváth and Kis [5] and a set partitioning-based mathematical model, where most vehicle-specific activities can be integrated based on the desired constraints is presented in [6]. This model is solved using a column generation approach. Békési and Nagy [7] presented a model to automatically calculate approximately optimal vehicle and driver schedules. For a given list of trips and considering company specific requirements and parameters in compliance with regulations. 


\subsection{P-Graph Methodology and Approaches}

The solution of vehicle scheduling problems are mainly based on the mathematical programming models as discussed in the previous section. During the generation of the mathematical programming models, directed graphs are often used to support certain steps. These directed graphs themselves usually correspond to the execution sequences only, in other words, they relate to the precedence order. All other pieces of information are hidden within the attributes of the nodes and edges of the graphs. These pieces of hidden information then appear within the variables and constraints of the mathematical programming models.

Another possible approach is to exploit the unique features of the structure of the problem at the earliest possible stage of the solution; this approach is followed in the present paper also. Conventional directed graphs are suitable for representing and analyzing processes, but they are not suitable for the synthesis step, see Friedler et al. [8].

The p-graph methodology has its origin in the early 90s, when Friedler et al. [8] first applied specially constructed directed bigraphs to depict and solve chemical engineering processes. In a chemical process, operating units transform their input materials to output materials by transforming the quality and quantity of the materials under consideration. To unambiguously represent this transformation, materials and operating units are represented by two separate types of vertices of the p-graph, while the interconnections are described by the arcs of the graph, i.e. arcs represent whether a material is consumed or produced by an operating unit. With the focus on this representation, certain combinatorial properties were formalized, i.e. a set of axioms was constructed. The first axiom states, for example, that every product should be represented in the p-graph. This set of axioms express the necessary and sufficient combinatorial properties to which a feasible process structure should confirm. In other words, based on these axioms the set of potentially feasible solution structure can be generated and conversely no other structures need be considered when the potentially feasible solutions are sought. Further, the maximal structure is defined as the union of all feasible solution structures. Please note that this maximal structure is of outmost importance in the p-graph methodology. Generally speaking, the maximal structure is similar to a "super-structure," however this later does not have a formal definition. With the focus on the underlying structure generation of the problem which serves as the basis of the mathematical programming model to be solved, all feasible solution structures can be enumerated algorithmically and listed according to their related results, see Friedler et al. [9]. Therefore, should a feasible solution be found by any method for the synthesis problem considered, then it is certain that this very solution is a subset of the maximal structure and further, it can be enumerated by the p-graph methodology. And conversely, no other solutions should be considered but only those enumerated by the p-graph methodology. 
Kovács et al. [10] explored fundamental structural properties of separation networks and studied the validity of the applied mathematical programming models specifically with respect to redundancy; classes of separation network synthesis problems were also studied extensively. They demonstrated that the synthesis step is critical when optimal separation networks are to be sought.

Sanmarti et al. [11] applied p-graphs to solve the scheduling of multipurpose batch plants. Süle et al [12] proposed the solution of supply chains with limitations as well as uncertainties of the renewable resources, i.e. the overall reliability of raw material availability is also considered when generating the n-most profitable supply chain alternatives. Tick et al. [13] applied p-graph methodology to business process modelling, giving both a nominal cost and an extended cost to each operating unit, while taking into consideration that only a limited number of operating units has the extended cost and the others have the nominal cost. A branch and bound based solution algorithm was presented together with a polynomial time dynamic programming algorithm for special problems. Vincze et al. [14] transformed CPM problems to p-graphs to manage the situation of alternative tasks and solutions within one step. Several corresponding mathematical programming models were given with illustrations how alternative cases appear in the structure. Later, Ercsey [15] solved a Hungarian clothing manufacturer's problem with p-graphs. Here, alternatives specified by mainly financial necessities as well as human resource constraints were managed within the proposed model.

An extension of the P-graph approach for multi-period process network synthesis is proposed by Tan and Aviso [16]. They offer a modification of the original p-graph approach to enable partial load operational lower limit for process units to be considered via the addition of fictitious streams. Tan et al. [17] combined p-graphs and Monte Carlo simulation approach to plan carbon management networks, generalized systems for minimizing emissions of $\mathrm{CO}_{2}$. Their target was to identify robust and near optimal carbon management networks, which can be achieved based on the optimal and near optimal solutions generated by the p-graph methodology.

Cabezas et al. [18] considered sustainable process systems and supply chains in their review article. They thoroughly investigated the usability of the p-graph methodology as well as the connections towards other methods. They also illustrated the advantages of the potential application of p-graphs from the feasible structures point of view. Fan et al. [19] used p-graphs as a decision support tool to develop an integrated design of waste management systems in support of a Circular Economy. They considered a number of case studies of municipal solid waste compositions based on different country income levels, identified the most suitable treatment approach as well as the near optimal solutions in order to deal with the trade-offs between conflicting objectives that are difficult to monetize. 
Recently, Bartos and Bertók [20] used p-graphs to determine the optimal load of automotive and electronic production lines where the assembly requires various components and significant number of human resources. Besides the algorithmic generation of the mathematical programming model and afterwards its resultant optimal solution, p-graphs here support the visual definition of the task to employee allocations. Bertók and Bartos [21] recently proposed an optimization method for energy allocation of renewable energy sources and storage considering different priorities of the producers, storage and consumers. They extended the original p-graph methodology to multi-period direction as well as to the direction where targets and intermediate entities cannot be accumulated.

König and Bertók [22] applied the p-graphs to freight transportation, and presented an algorithmic method which is capable to involve and enumerate all feasible scenarios, when analyzing the conditions of the contracts in case of uncertainty, i.e. which solution costs less or offer more flexibility. Bárány et al. [23] proposed a p-graph based method for minimizing cost and emission for vehicle assignment problems. There, the assignment problem is transformed into a p-graph, which provides the structural model and the basis for the solution. The approach includes the maximal structure generation, which further serves as the input for the generation and solution of the mathematical programming model. At the end, the optimal and a finite number of $n$-best sub-optimal networks in the ranked order are given.

Nagy et al. [24] already investigated bus transport problems within the p-graph methodology. There, the scenario considers various periods during the day, when the launching density of the buses can be considered to be the same; i.e. the scenario with a periodic timetable. With the focus on the synthesis step, the material type and the operating unit type vertices of the p-graph were specified and explained. Maximal structures for i) a one period and one bus without driver change problem, for ii) a one period and one bus with driver change problem and for iii) a two period and one bus without driver change problem were given together with a corresponding MILP / NLP mathematical programming model. From the maximal structure all feasible solution structures are generated. The corresponding mathematical programming model that suits to the periodic bus transport problem is explained in detail. This MILP / NLP model has to be solved for each generated solution structure, and the result prepares the generation of the launching table of the buses. In other words, bus transport problems with periods within the timetable are suggested to be solved in [24].

The current work expands the scenario of [24], namely when the timetable of the buses has no periods, but arbitrary individual launching times are defined is considered as the basic scenario of the present paper. This modification points towards a more general practical situation, nevertheless it has a fundamental effect on the p-graph elements, and therefore on the maximal structure, as well as on the related mathematical programming model that needs to be considered. Moreover, compared to the other methods cited in Section 2.1 where expansive mathematical 
programming models have to be solved often arduously, here the corresponding mathematical programming model can be solved even with publicly available solvers.

\subsection{Problem Definition}

Exploiting the above mentioned antecedents and results the following bus transport problem is discussed and solved hereinafter based on the p-graph methodology. Let the timetable of the buses with individual launching times be given, i.e. the starting times within the timetable can be arbitrarily given, and no periods can be determined within the timetable. The main goal is to determine the specific bus transport schedule that meets the given timetable. Obviously, besides this main goal, other viewpoints may also be considered and to be optimized, for example minimum fuel consumption of the buses considered, etc.

Let us consider the situation, where the departure station and the terminal station are given. Let the driving time of the turn also be given to each determined starting time. Obviously, the bus fleet is also given together with the number of different buses, the number of drivers etc. together with the parameters of the stance, secession. Obviously, labor standards of the drivers have to be considered and therefore, also be given in advance.

In summary, the following characteristics are considered:

- Bus route, containing information about the departure station and terminal station. Nametag, other stations etc. in connection with the bus route is also available.

- Bus turn, containing information about the bus route, and specific time information etc. The followings are considered hereinafter:

- Bus launching times: $\mathrm{P}_{1}, \mathrm{P}_{2}, \ldots, \mathrm{P}_{\mathrm{N}}$, (the launching time of the first bus: $\mathrm{P}_{1}$, the launching time of the last bus: $\mathrm{P}_{\mathrm{N}}$ )

- $\quad$ Time required to perform the turn, $\mathrm{T}_{1}, \mathrm{~T}_{2}, \ldots, \mathrm{T}_{\mathrm{N}}$

- The arrival time of the given bus, $\mathrm{p}_{1}, \mathrm{p}_{2}, \ldots, \mathrm{p}_{\mathrm{N}}$, can be calculated from the launching time and the time required for the turn.

- Activities, containing information about rest, stance etc. both concerning duration, and whether the activity has to be considered within the working hours and driving hours.

- $\quad$ Time for the rest period, in minutes, RT

- Time for changeover, in minutes, AT

- $\quad$ Time for driver change, in minutes, HT 
- $\quad$ Time for discharge, in minutes, LT

- $\quad$ Time for stance, in minutes, ST

- $\quad$ Time for entering the garage, in minutes, GT

- Labour standards, containing information about its type, working hours etc. The type can be normal or split:

- $\quad$ Minimum working hours, in minutes, NWH

- Maximum working hours, in minutes, XWH

- Maximum hours until rest

- Fleet, containing information about the available buses

- $\quad$ Number of buses, B

- Number of drivers, D

Please note that the present problem definition does not depend on the bus line itself and therefore may consider multiple bus lines together. It is however important that the bus lines have the same departure and terminal stations and their routes and turns are explicitly specified and known as above. For example, both bus line $A$ and bus line $B$ be explicitly specified as above with a sole launch per bus line, with bus line $A$ having an earlier launching time. Then, the data corresponding to the first launch, i.e. $P_{1}, p_{1}$ and $T_{1}$ will refer to the bus line $A$, while the second launch, i.e. $P_{2}, p_{2}$ and $T_{2}$ will refer to the bus line $B$.

\section{Results}

\subsection{Solution Framework}

The bus transport problem specified in the previous section is proposed to be solved by an algorithmic method as follows. First, the structural model, namely the maximal structure of the bus transport problem is generated and the parameters of the arcs and nodes are set. Please note that many characteristics of the problem can already be considered with the exact formulation of the structural model. Second, the corresponding mathematical programming model is generated and solved. The solution of the model serves as the solution of the bus transport problem with arbitrary bus launching times. Details are given in the subsequent chapters. The solution framework is given in Figure 1 with the emphasis on the synthesis step. 


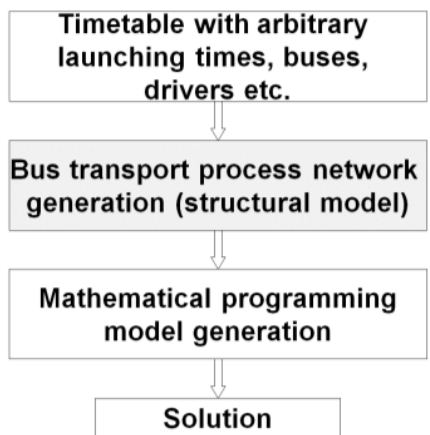

Figure 1

Solution framework of the bus transport synthesis problem with arbitrary launching times

\subsection{Maximal Structure}

The key elements of the maximal structure of the bus transport synthesis problem with arbitrary launching times are given in Figure 2. Please note that the subgraph represented by red color have to be considered within the maximal structure of the bus transport synthesis problem with arbitrary launching times as many times as many departure times are given within the timetable. Here, a bus and a driver after performs a turn. After the turn, the driver can go back to the garage or have a rest or have no rest. Drivers change is also acceptable. It is also visible, that after the completion of the turn the bus can perform further turns.

On this p-graph, buses and drivers are given as nodes of the raw material type, while garage and work shift end for the driver are given as nodes of the product type. Intermediate material type nodes represent departures and arrivals as follows: the arbitrary launches of the buses given in the timetable are represented by the nodes $P_{l}, P_{n}, P_{k}, P_{N}$, while the corresponding arrivals are represented by the nodes $p_{n}$, and $p_{N}$. Intermediate material type node with the label $M \_n$ represents the situation either without rest period after the completed turn, or with the rest period following the turn, or the situation when drivers change happen after the turn.

The operating unit type nodes represent the various activities, namely stance, turn, changeover, secession, no rest, rest and driver change. Since no rest, rest and driver change are parallel activities, should only one of them be within the solution of the $n$-th turn, the other two have to be omitted. Please observe that the size of the maximal structure given in Figure 2 depends on the number of individual launches; it is specified in Table 1 how the approach scales with the problem size.

It is important to mention that more complex bus driver scheduling problems may have the same or different maximal structures, depending on the problem. For example, real cases corresponding to the fact that only one or a maximum 
number of buses may be at the departure station at a time, since there is no more place for other buses for example, has no effect on the given maximal structure, this limitation has to be controlled within the subsequent mathematical programming model. Other cases, for example when driver change is permitted in between the departure and terminal stations of the turn corresponds to an altered maximal structure. This case is depicted in Figure 3, where the effect of this new condition is highlighted with violet color. Here, an additional operating unit type node representing the activity of the turn and the driver change together have the labels $T D C_{-} i$. It is worth mentioning that since there are constraints on the possible working hours of the drivers, it is not realistic to perform a driver change at the last turn.

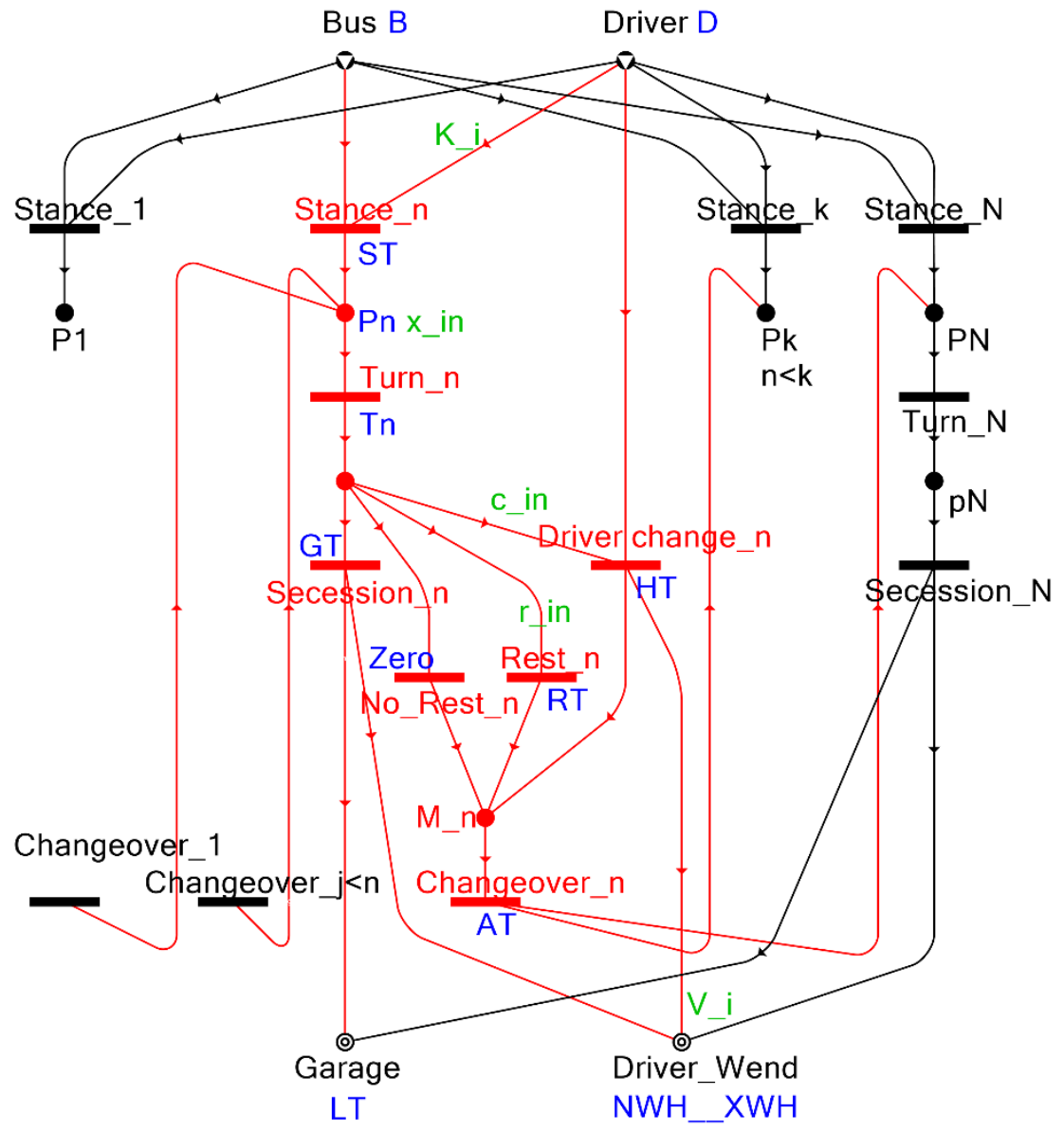

Figure 2

Key elements of the maximal structure of the bus transport synthesis problem with arbitrary launching times, the $n$-th launch is highlighted 


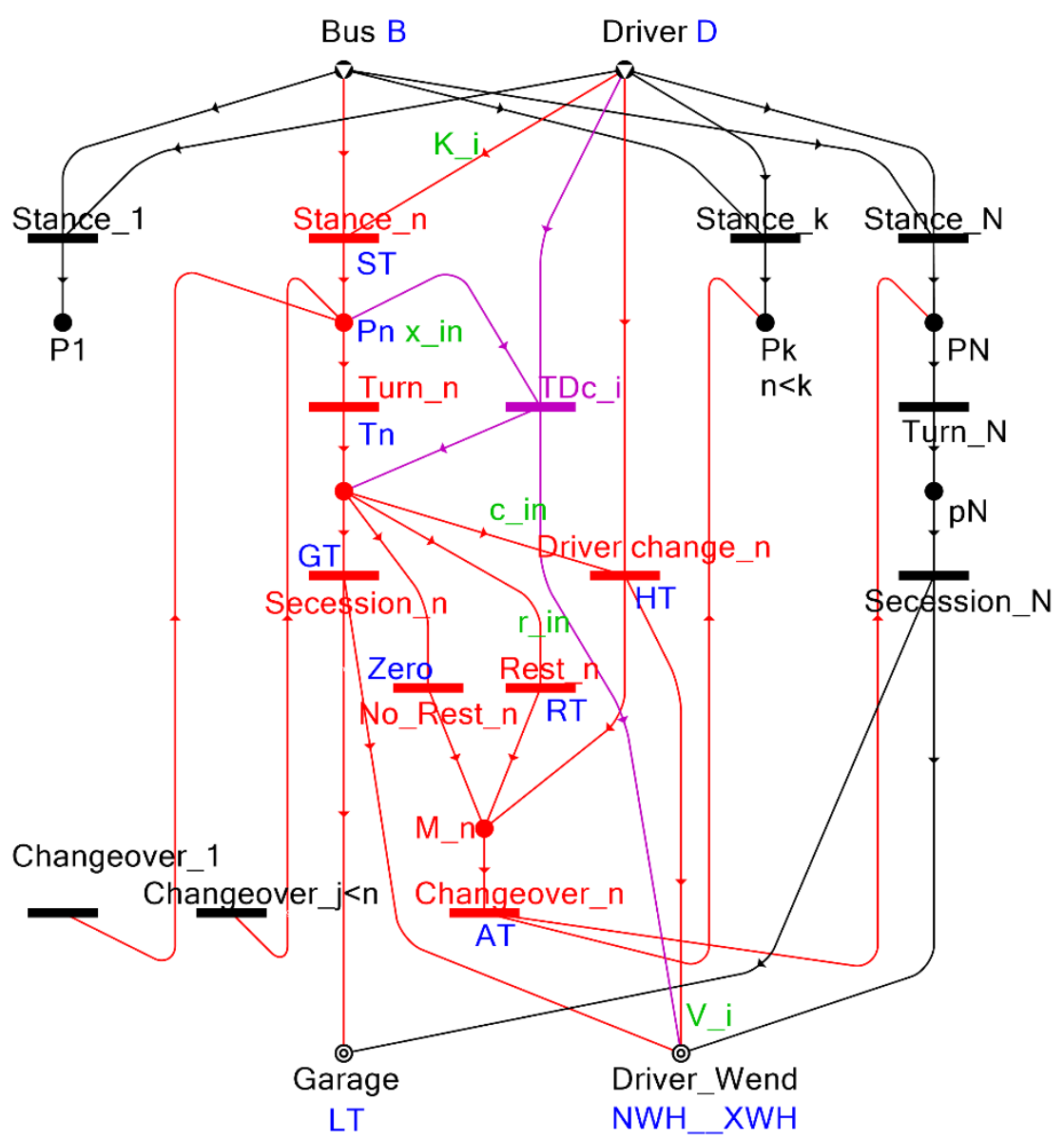

Figure 3

Key elements of the maximal structure of the bus transport synthesis problem with arbitrary launching times where driver change is permitted in between the departure and terminal stations of the turn, the $n$-th launch is highlighted

Table 1

Size of the maximal structure given in Figure 2, where $n$ is the number of individual launches

\begin{tabular}{|c|c|}
\hline Graph element & Size of the maximal structure \\
\hline Edges & $|E|=2 n+6 n+\frac{n(n-1)}{2}+9(n-1)$ \\
& $=\frac{1}{2} n^{2}+\left(17-\frac{1}{2}\right) n-9$ \\
\hline Operating unit type nodes & $|O|=7 n-4$ \\
\hline Material type nodes & $|M|=3 n-1+4$ \\
\hline
\end{tabular}




\subsection{Mathematical Programming Model}

In the previous chapter illustrated how the maximal structure of the bus transport process network, with arbitrary launches, is developed. Based on the maximal structure given in Figure 2 the following mathematical programming model is given. Please note that the parameters of the problem are highlighted with blue, and variables are highlighted with green color in Figure 2.

Let binary variable $d_{i}$ denote whether the $i$-th driver takes part in the solution or not. Further, let binary variable $x_{i n}$ denote whether the $i$-th driver in the $n$-th bus turn departed or not. Let binary variable $r_{i n}$ denote whether the $i$-th driver after completion of the $n$-th bus turn takes its rest or not. Let binary variable $c_{i n}$ denote whether the $i$-th driver after completion of the $n$-th bus turn finishes his work shift and hands the bus over to another driver, i.e. a driver change activity is performed. Moreover, let variable $K_{i}$ corresponds to the start, while $V_{i}$ correspond to the end of the working hours of the $i$-th driver.

\section{Constraints subject to the labor standards}

The labor standards regarding the minimal and maximal length of the working hours have to be considered. Please see equation (1) and (2). It also has to be noted that the duration of the rest cannot be considered as working hours.

$\begin{array}{ll}\forall i=\{1,2, \ldots, D\} & V_{i}-K_{i} \leq X W H+\sum_{n=1}^{N} r_{i n} * R T \\ \forall i=\{1,2, \ldots, D\} & V_{i}-K_{i} \geq N W H-\sum_{n=1}^{N} r_{i n} * R T\end{array}$

Constraints subject to the start and end of the $i$-th driver's work shift

$\forall i=\{1,2, \ldots, D\} \forall n=\{1,2, \ldots, \mathrm{N}\}$

$V_{i} \geq P_{n}+T_{n}+c_{i n} * H T+\left(1-c_{i n}\right) * G T+L T-\left(1-x_{i n}\right) * M$

Equation (3) corresponds to the fact that the end of the work shift of the $i$-th driver has to be later than the arrival time of the given turn plus discharge and entering the garage. This inequality is a dynamic one, it has to be regularly updated, obviously, the final constraint cannot be known in advance.

$\forall i=\{1,2, \ldots, D\} \quad \forall n=\{1,2, \ldots, \mathrm{N}\} \quad K_{i} \leq P_{n}-S T+\left(1-x_{i n}\right) * M$

Equation (4) is similar to equation (3), but from the other direction, i.e. dynamic equation, regularly updated backwards. The launching time of the last bus turn is known. The start of the work shift of the $i$-th driver is set accordingly to the earliest bus turn performed by the driver.

\section{Constraints subject to the bus turns}

Equation (5) corresponds to the fact that the bus turns have to be performed by one of the drivers, while less than one is not sufficient and more drivers are superfluous. 
$\forall n=\{1,2, \ldots, N\} \quad \sum_{i=1}^{D} x_{i n}=1$

Equation (6) corresponds to the fact that the bus driver cannot start a new bus turn before successfully finishing and fully returning from his previous bus turn.

$\forall i=\{1,2, \ldots, D\} \quad \forall n=\{1,2, \ldots, N-1\} \quad \forall k=\{n+1, n+2, \ldots, N\}$
$x_{i k} * P_{k} \geq x_{i n} *\left(P_{n}+T_{n}+A T\right)+r_{i n} * R T+c_{i n} * H T$

\section{Constraints subject to the rest period of the drivers}

The binary variable $r_{\text {in }}$ denotes whether the $i$-th driver after completion of the $n$-th bus turn takes its rest or not, namely it is 1 in case there is a rest period and 0 otherwise.

Should the working hours exceed 4 hours, then the driver has to have a rest period, it is specified by equation (7).

$\forall i=\{1,2, \ldots, D\} \forall n=\{1,2, \ldots, N\}$

$P_{n}-K_{i}-240 *\left(\sum_{n=1}^{N}\left(r_{i N}\right)\right) \leq 240+\left(1-x_{i n}\right) * M$

The rest period cannot be within the last working hour of the driver; it is specified by equation (8).

$\forall i=\{1,2, \ldots, D\} \quad \forall n=\{1,2, \ldots, N\} \quad V_{i}-P_{n} * r_{i n} \geq 60$

The rest period cannot start within the first working hour of the driver, it is specified by equation (9), where $M$ is a large constant, $M>1440$, for example 10000 .

$\forall i=\{1,2, \ldots, D\} \quad \forall n=\{1,2, \ldots, N\} \quad P_{n} * r_{i n}-K_{i} \geq 60+\left(r_{i n}-1\right) * M$

\section{Constraints subject to the resources}

Should a driver change activity happen, the original driver hands over the bus to the new driver and finishes his work shift, it is specified by equation (10); while the constraints for the drivers is specified in equation (11).

$\sum_{i=1}^{D} d_{i}-\sum_{i=1}^{D} \sum_{n=1}^{N} c_{i n} \leq B$

$\forall i=\{1,2, \ldots, D\} \quad \frac{1}{N} * \sum_{n=1}^{N} x_{i n} \leq d_{i} \leq \sum_{n=1}^{N} x_{i n}$

\section{Cost function}

The cost function considered is to minimize all unwanted waiting time of the bus drivers.

$$
\sum_{i=1}^{D}\left(\left(V_{i}-K_{i}\right)-\sum_{n=1}^{N} r_{i n} * R T\right) \rightarrow \min
$$




\subsection{Example}

As part of a daily work to optimally handle bus transport problems, from the traffic point of view a practical example was received from the Budapest Transport Corporation as a public transport service company. The example is based on a real situation and the problem is considered to be typical and of medium difficulty there. For the illustration of the present method only the key parameters of the problem are considered as follows. There is a single depot, the route length is $5.4 \mathrm{~km}$, there are 13 stations along the route, there are 178 bus turns launched within a day, driver change is allowed at the departure station, there are 6 buses and 11 drivers available, rest period can be given only at the departure station. First, the maximal structure of the bus transport process network with arbitrary bus launching times problem was determined based on Figure 2. It is worth mentioning that the bus service company does not consider the situation practical where driver change is permitted in between the departure station and the terminal station practical for this line, therefore the maximal structure depicted on Figure 3 was not considered further. With exploiting the advantages of this structural representation, the corresponding mathematical programming model was generated as detailed in Section 3.3. The size of this mathematical programming model can be handled with relative ease, since based on the previous steps the number of variables in the resultant simplex table is limited. An MPS file was generated automatically, which is a column oriented text format storing linear programming problems and which is supported by a large number of solvers. For the particular case, CPLEX and FICO Xpress were used, as a publicly available NEOS solvers. The solvers were controlled with a time limit.

Please note that the mathematical programming model for the particular problem has 5885 binary variables, plus 22 non negative, real variables; furthermore, the model has 183296 constraints. Should more detailed regulations on the labor standards, work shifts etc. be considered, these appear as additional constraints within the model only, and does not have a significant effect on the scale of the problem. The minimum number of drivers necessary to solve this problem is 9 , with less drivers the problem is infeasible. The total waiting time of the drivers is 17 hour and 57 minutes, namely the time when the drivers do not perform any useful activities neither rest; in other words, each driver has approximately 2 hours of unwanted waiting time, which is already a better solution than the current solution applied in the everyday life at the transportation company. The solution data is summarized in Table 2.

Table 2

Solution details of the example

\begin{tabular}{|c|c|c|c|}
\hline Driver & Start & End & $\begin{array}{c}\text { Working hours } \\
\text { (minutes) }\end{array}$ \\
\hline 1 & $04: 58$ & $15: 18$ & 620 \\
\hline 2 & $05: 18$ & $09: 42$ & 264 \\
\hline
\end{tabular}




\begin{tabular}{|l|l|l|l|}
3 & $06: 47$ & $15: 54$ & 547 \\
\hline 4 & $07: 17$ & $17: 44$ & 627 \\
\hline 5 & $09: 25$ & $19: 34$ & 609 \\
\hline 6 & $15: 17$ & $23: 55$ & 518 \\
\hline 7 & $13: 49$ & $19: 04$ & 315 \\
\hline 8 & $15: 37$ & $23: 35$ & 478 \\
\hline 9 & $17: 17$ & $22: 55$ & 338 \\
\hline
\end{tabular}

\section{Conclusions}

Herein, bus transport problems, with arbitrary launching times, were discussed. An approach based on the p-graph methodology was proposed to concentrate on the synthesis step of the problem. In contrast to other general purpose solution frameworks, this approach focuses on the pure structure of the system. The key elements of the maximal structure of the problem were detailed. A corresponding mathematical programming model was presented, that suits the particular maximal structure. This model had the advantage of being limited in the number of variables and constraints and therefore, publicly available solvers were capable of generating results and real solutions of industrial problems. A practical example with medium problem difficulty, available at a public transport company, illustrated that the solution method proposed is effective.

\section{Acknowledgement}

The authors greatly acknowledge the valuable contribution of Dávid Vizi and Optin Ltd.

\section{References}

[1] Bodin L, Golden B, Assad A and Ball M. Routing and Scheduling of Vehicles and Crews: The State of the Art, Computers and Operations Research, 10, 63-211, 1983

[2] Kliewer N, Mellouli T and Suhl L. A time-space network based exact optimization model for multi-depot bus scheduling, European Journal of Operational Research, 175, 1616-1627, 2006

[3] Dávid B and Krész M. Application Oriented Variable Fixing Methods for the Multiple Depot Vehicle Scheduling Problem, Acta Cybernetica, 21(1), 53-73, 2013

[4] Tóth A and Krész M. An efficient solution approach for real-world scheduling problems in urban bus transportation, Central European Journal of Operations Research, 21(1), 75-94, 2013

[5] Horváth $\mathrm{M}$ and Kis $\mathrm{T}$. Computing strong lower and upper bounds for the integrated multiple-depot vehicle and crew scheduling problem with branch-and-price, Central European Journal of Operations Research, 27(1), 39-67, 2019 
[6] Békési J, Dávid B and Krész M. Integrated Vehicle Scheduling and Vehicle Assignment, Acta Cybernetica, 23(3), 783-800, 2018

[7] Békési J and Nagy A (2020) Combined Vehicle and Driver Scheduling with Fuel Consumption and Parking Constraints: a Case Study. Acta Polytechnica Hungarica, Vol. 17, No. 7, 2020, DOI: 10.12700/APH.17.7.2020.7.3

[8] Friedler F, Tarjan K, Huang Y, Fan LT. Graph-theoretic approach to process synthesis: axioms and theorems. Chem Eng Sci 47(8), 1973-1988, 1992

[9] Friedler F, Varga J, Fan LT. Decision-mapping: a tool for consistent and complete decisions in process synthesis. Chem Eng Sci 50(11), 1755-1768, 1995

[10] Kovacs Z, Ercsey Z, Friedler F and Fan LT. Redundancy in a separationnetwork. Hungarian Journal of Industry and Chemistry 26(3), 213-219, 1998

[11] Sanmarti E, Puigjaner L, Holczinger $\mathrm{T}$ and Friedler F. Combinatorial framework for effective scheduling of multipurpose batch plants. Aiche Journal 48(11), 2557-2570, 2002

[12] Sule Z, Bertok B, Friedler F and Fan LT. Optimal design of supply chains by P-graph framework under uncertainties. Chem Eng 25: 453-458, 2011

[13] Tick J, Imreh C and Kovács Z. Business Process Modeling and the Robust PNS Problem. Acta Polytechnica Hungarica, 10(6), 193-204, 2013

[14] Vincze, N, Ercsey Z, Kovács T, Tick J, and Kovács Z. Process Network Solution of Extended CPM Problems with Alternatives, Acta Polytechnica Hungarica, 13(3), 101-117, 2016

[15] Ercsey Z. Process network solution of a clothing manufacturer's problem. Pollack Periodica 12(1), 59-67, 2017

[16] Tan RR and Aviso KB. An extended P-graph approach to process network synthesis for multi-period operations. Comput Chem Eng 85, 40-42, 2016

[17] Tan, RR, Aviso KB, and Foo, DCY. P-graph and Monte Carlo simulation Approach to planning carbon management networks, Computers \& Chemical Engineering, 106, 872-882, 2017

[18] Cabezas H, Argoti A, Friedler F, Mizsey P and Pimentel J. Design and Engineering of Sustainable Process Systems and Supply Chains by the PGraph Framework. Environmental Progress \& Sustainable Energy 37(2) 624-636, 2018

[19] Fan YV, Klemeš JJ, Walmsley TG and Bertók B. Implementing Circular Economy in municipal solid waste treatment system using P-graph. Science of the Total Environment 701, 134652, 2020 
[20] Bartos A and Bertok B. Production line balancing by P-graphs. Optim Eng $8(6), 1-18,2019$

[21] Bertók B and Bartos A. Renewable energy storage and distribution scheduling for microgrids by exploiting recent developments in process network synthesis. Journal of Cleaner Production 244, 118520, 2020

[22] König É and Bertók B. Process graph approach for two-stage decision making: Transportation contracts. Computers and Chemical Engineering $121,1-11,2019$

[23] Bárány B, Bertók B, Kovács Z, Friedler F and Fan LT. Solving vehicle assignment problems by process-network synthesisto minimize cost and environmental impact of transportation. Clean Technol Environ Policy 13(4):637-642, 2011

[24] Nagy A, Ercsey Z, Tick J and Kovács, Z. Bus Transport Process Network Synthesis Acta Polytechnica Hungarica 16(7) 25-43, 2019 